\title{
Common sense or evidence: an optimal place for right and left ventricular leads?
}

\author{
I. Eli Ovsyshcher
}

Received: 25 February 2015 / Accepted: 6 March 2015 / Published online: 16 April 2015

(C) Springer Science+Business Media New York 2015

\begin{abstract}
"We do not see things as they are, we see them as we are"

Talmud: Tractate Berakoth, Folio 55b, Ed. Rabbi Dr. I. Epstein.

"All true knowledge contradicts common sense."

Mandell Creighton, Cambridge professor of British his-

tory and a Bishop of the Church of England.
\end{abstract}

Increased understanding and recognition of the chronic adverse effects of right ventricular (RV) pacing (mainly apical) in the last decade has stimulated interest in strategies for its attenuation. These effects, left ventricular (LV) remodeling, progressive LV systolic dysfunction, and increased risk in heart failure (HF), may be due to dyssynchronous myocardial electrical activation that bypasses the native cardiac conduction, manifesting itself on the surface ECG as QRS widening.

Accordingly, a number of different pacing algorithms have been developed that reduce the degree of RV pacing. Despite expectations regarding the physiological rationale of DDD pacing with algorithms minimizing RV pacing, the results of the ANSWER study [1] as well as three other previous large randomized trials (DANPACE, PreFERMVP, and MINE RVA) fail to demonstrate an overwhelming advantage of pacing minimization approaches over conventional pacing [1]. This lack of significant clinical effect is contrary to expectations and common sense. Explanations for this obvious contradiction have been discussed [1].

I. E. Ovsyshcher

Cardiology, Faculty of Health Sciences, Ben-Gurion University,

Beersheva, Israel

I. E. Ovsyshcher $(\bowtie)$

Cardiology, Soroka University Medical Center, P.O. Box 151,

Beersheva 84101, Israel

e-mail: eliovsy@bgu.ac.il
An additional approach to reducing damage of RV pacing (RVP) was the selection of a new or alternative site such as His bundle/para Hisian tissues or various "septal" positions. The most interesting and feasible location is the upper part of the septum because of its proximity to the His bundle which renders it the earliest zone in heart depolarization [2]. Consequently, the QRS here is narrower than from the apex. Moreover, this part of the septum is trabeculated and suitable for active fixation leads. Speculations that pacing from this rather than from other areas would achieve better heart contraction, especially from the apex, is a feasible solution for many implanters and they routinely use this approach hoping to decrease the future risks of pacing-induced HF [2]. Furthermore, some enthusiasts recommend RV septal pacing as a substitute for CRT. Subsequently, the recent EHRA/HRS expert consensus noted regarding RV lead location: "septal pacing may be preferred in conventional pacemakers"[3]. However, experimental and clinical studies demonstrated that various RV pacing sites did not recruit the His-Purkinje system [2, 4]. The majority of LV activation instead occurred via slow and dyssynchronous direct myocardial to myocardial cell conduction and that in terms of mechanical dyssynchrony, hemodynamic and electrical parameters septal pacing was not superior to $\mathrm{RV}$ apical pacing $[2,4]$. A recent cross-sectional multicenter study demonstrated that for pacing in RV, there is no better alternative to apex [5]. The systematic review and metaanalysis of all randomized controlled studies in DDD patients compared to RV alternative sites pacing versus apical provided inconclusive results with respect to exercise capacity, functional class, quality of life, and survival [6]. Additionally, the conclusion of the recently published PROTECT-PACE study demonstrated that in patients with AV block and preserved LV function requiring a high percentage of RVP, septal pacing does not provide a protective effect on LV function over RV apical pacing and a significantly greater time was required to 
place the lead in the septal position [7]. The results of this study as well as previous ones [6] are counterintuitive. There are some explanations for this apparent challenge.

1. Pacing from any RV site may be harmful, and electrophysiological differences are not clinically significant among sites; therefore, pacing from alternative sites, including septum, cannot provide physiological pacing, and difference between sites is nonsignificant in terms of clinical outcomes [2, 4-8].

2. Differing composition of patient populations in studies explored various sites of RV pacing, e.g., SND versus AV block.

3. Inadvertent incorrect positioning of the RV lead.

The second explanation needs clarification. Patients with depressed LVEF and narrow QRS (i.e., without electrical dyssynchrony) may particularly be harmed by RVP-induced dyssynchrony. However, those with LBBB or wide QRS, due to nonspecific intraventricular conduction defect (IVCD) and already compromised electromechanical activation, may experience less damage by RV pacing because this preceding dyssynchrony can extenuate the harmful effects of RVP ( $20 \%$ of the pacemaker population had LBBB or IVCD) [1]. Thus, whereas in patients with LBBB, RV pacing provides a shorter electromechanical delay, which correlates with an improvement in LV function, the converse effect is observed in patients without LBBB [9, 10]. This inconsistent impact of RV pacing explains observations that in CRT patients, RVP is a positive part of the resynchronization process and RV lead position has no effect on clinical outcomes in CRT patients [3].

The former explanation-inadvertent incorrect positioning of the RV lead-supported by solid evidence in the study of Jackson II LR et al. published in this issue of the journal [11]. In the study, 25 experienced boardcertified electrophysiologists interpreted RV lead position using standard $\mathrm{x}$-ray views which were then compared to $\mathrm{CT}$ as reference (experienced board-certified cardiothoracic radiologist). Three-dimensional CT datasets verified RV lead position in the apex in $37 \%$ of patients, in the septum in $21 \%$ and most frequently in the anterior-free wall-in $42 \%$ of patients. The authors stressed that inadvertent RV lead positioning on the anterior-free wall is associated not only with a higher risk of procedure related complications but with both worsening of RV and LV dyssynchrony and their function compared to lead placement on the septum or apex of the RV.

The agreement between x-ray and CT lead position (accuracy) was recorded only in $37 \%$ with $64 \%$ reproducibility. This data can explain controversial results among various studies comparing RV apical pacing with alternative approaches for placement RV lead. This data also can, at least partly, explain results among various studies on clinical outcomes of RV pacing minimization [1].

It is of interest to note that the situation was no better situation for identifying the LV lead position in CRT patients, with respect to accuracy and reproducibility.

These findings have important implications not only for the implantation of pacing and defibrillation systems but are especially important because they partially explain paradoxical results of studies comparing RV apical pacing with RV alternative sites as well as studies examining the importance of $\mathrm{LV}$ lead position for respond to CRT.

The results reported by Jackson II LR et al. again emphasize the consequences of imprecise placement of ventricular leads. The study presents solid evidence that standard $\mathrm{x}$-ray is an inaccurate method for placement of ventricular leads and consequently inadequate for optimal treatment of both bradyarrhythmias and HF.

To establish the most favorable ventricular lead position and its real influence on clinical outcomes, the results of the study should be applied.

Currently, available data is too controversial and not sufficiently scientific regarding optimal ventricular lead placement, and for future studies using multi-dimensional imaging techniques for evaluation, its positions are warranted.

\section{References}

1. Bogdan, S., \& Glikson, M. (2015). Physiological pacing: a moving target? (Editorial to Stockburger M, Boveda S, Moreno J, Da Costa A, Hatala R, Brachmann J, et al. Eur Heart J. 2015;36:151-7). European Heart Journal, 36, 141-142.

2. Pang, B. J., Kumar, S., Tacey, M. A., \& Mond, H. G. (2014). Capturing the His-Purkinje system is not possible from conventional right ventricular apical and nonapical pacing sites. PACE, 37, 724730 .

3. European Heart Rhythm Association, European Society of Cardiology, Heart Rhythm Society, Heart Failure Society of America, American Society of Echocardiography, American Heart Association, European Association of Echocardiography, Heart Failure Association, Daubert, J. C., Saxon, L., Adamson, P. B., Auricchio, A., Berger, R. D., Beshai, J. F., Breithard, O., Brignole, M., Cleland, J., Delurgio, D. B., Dickstein, K., Exner, D. V., Gold, M., Grimm, R. A., Hayes, D. L., Israel, C., Leclercq, C., Linde, C., Lindenfeld, J., Merkely, B., Mont, L., Murgatroyd, F., Prinzen, F., Saba, S. F., Shinbane, J. S., Singh, J., Tang, A. S., Vardas, P. E., Wilkoff, B. L., \& Zamorano, J. L. (2012). 2012 EHRA/HRS expert consensus statement on cardiac resynchronization therapy in heart failure: implant and follow-up recommendations and management. Heart Rhythm, 9, 1524-76.

4. Mills, R. W., Cornelussen, R. N., Mulligan, L. J., Strik, M., Rademakers, L. M., Skadsberg, N. D., van Hunnik, A., Kuiper, M., Lampert, A., Delhaas, T., \& Prinzen, F. W. (2009). Circulation. Arrhythmia and Electrophysiology, 2(5), 571-9.

5. Janoušek, J., van Geldorp, I. E., Krupičková, S., Rosenthal, E., Nugent, K., Tomaske, M., Früh, A., Elders, J., Hiippala, A., Kerst, G., Gebauer, R. A., Kubuš, P., Frias, P., Gabbarini, F., Clur, S. A., Nagel, B., Ganame, J., Papagiannis, J., Marek, J., Tisma-Dupanovic, 
S., Tsao, S., Nürnberg, J. H., Wren, C., Friedberg, M., de Guillebon, M., Volaufova, J., Prinzen, F. W., \& Delhaas, T. (2013). Working group for cardiac dysrhythmias and electrophysiology of the association for European pediatric cardiology. Circulation, 127(5), 613-23.

6. Shimony, A., Eisenberg, M. J., Filion, K. B., \& Amit, G. (2012). Beneficial effects of right ventricular non-apical vs. apical pacing: a systematic review and meta-analysis of randomized-controlled trials. Europace, 14, 81-91.

7. Kaye G. C., Linker N. J., Marwick T. H., Pollock L., Graham L., Pouliot E., Poloniecki J., Gammage M.; on behalf of the Protect-Pace trial investigators.(2014). Effect of right ventricular pacing lead site on left ventricular function in patients with high-grade atrioventricular block: results of the Protect-Pace study. Europe Heart Journal [Epub ahead of print]

8. ten Cate, T. J., Scheffer, M. G., Sutherland, G. R., Verzijlbergen, J. F., \& van Hemel, N. M. (2008). Right ventricular outflow and apical pacing comparably worsen the echocardiographic normal left ventricle. European Journal of Echocardiography, 9, 672-7.

9. Garrigue, S., Barold, S. S., Valli, N., Gencel, L., Jais, P., Haissaguerre, M., \& Clémenty, J. (1999). Effect of right ventricular pacing in patients with complete left bundle branch block. American Journal of Cardiology, 83, 600-4.

10. Varma, N. (2015). Left ventricular electrical activation during right ventricular pacing in heart failure patients with LBBB: visualization by electrocardiographic imaging and implications for cardiac resynchronization therapy. Journal of Electrocardiology, 48, 53-61.

11. Jackson II, L. P., Piccini Sr. J. P., Daubert J. P., Hurwitz L. M., Atwater B. D. Localization of pacing and defibrillator leads using standard X-ray views is frequently inaccurate and is not reproducible. Journal of Interventional Cardiac Electrophysiology. 